Trauma Berufskrankh $2006 \cdot 8$ [Suppl 1]: S49-S54 DOI 10.1007/s10039-006-1115-1

Online publiziert: 06. April 2006

๑) Springer Medizin Verlag 2006

B. Schewe $\cdot$ K. Weise

BG-Unfallklinik, Tübingen

\title{
Therapie von Knorpelschäden
}

\section{Was ist gesichert?}

In einer retrospektiven Studie über 31.515 Arthroskopien des Kniegelenks wurde in $63 \%$ der Fälle eine Knorpelläsion gefunden. Bei 19\% der Patienten lag ein viertgradiger Knorpelschaden vor, $5 \%$ dieser Patienten waren unter 40 Jahre alt [16]. In einer Studie mit 993 Arthroskopien wurde in $66 \%$ der Kniegelenke eine pathologische Veränderung des Knorpels berichtet. Bei 11\% lag ein vollschichtiger Knorpelschaden vor, und in $6 \%$ der Fälle war der Defekt über $2 \mathrm{~cm}^{2}$ groß [5]. In einer skandinavischen Arbeit mit 1000 Kniegelenkspiegelungen lag in $16 \%$ der Fälle ein chondraler oder osteochondraler Defekt vor [27]. Diese Untersuchungen deuten darauf hin, dass umschriebene Knorpelschäden des Kniegelenks in den meisten Altersgruppen ziemlich häufig sind.

Insgesamt sind in den meisten epidemiologischen Untersuchungen innerhalb der großen Gelenke für das Kniegelenk die höchsten Prävalenzraten für eine Arthrose beschrieben [18]. Man kann davon ausgehen, dass durch die demografische Entwicklung in den kommenden Jahren die Zahl der Arthrosepatienten noch weiter zunehmen und damit die soziökonomische Belastung steigen werden. Bereits heute belaufen sich die Kosten für die Arthrosebehandlung in Deutschland auf rund 10 Mrd. EUR jährlich [40].

\section{Spontanverlauf umschriebener Knorpeldefekte des Kniegelenks}

Bei frischen traumatischen Knorpelschäden oder einer Osteochondrosis dissecans findet man meist lokal umschrie- bene Defekte bei ansonsten intakter Knorpelumgebung. Handelt es sich um einen klinisch symptomatischen Defekt, findet man häufig einen begleitenden Gelenkerguss, eine Hypertrophie der Gelenkinnenhaut und/oder eine Tendinitis oder Bursitis des betroffenen Gelenks [19]. Während im weiteren Verlauf bei Kindern und Jugendlichen nicht selten eine Spontanheilung des Knorpelschadens beobachtet werden kann, stellt dies beim Erwachsenen eher die Ausnahme dar [15]. Trotzdem kann ein solcher auch nach dem Schluss der Wachstumsfugen klinisch stumm verlaufen. Selbst größere Defekte oder fortgeschrittene degenerative Veränderungen des Gelenkknorpels können mit geringer Symptomatik einhergehen [46]. Besonders bei kleineren Defekten kann auch nach längerer Verlaufszeit die Ausbildung einer Gonarthrose ausbleiben [44].

Auf der anderen Seite konnte in verschiedenen Studien gezeigt werden, dass das Arthroserisiko unabhängig vom Patientenalter mit zunehmender Größe eines primären Knorpelschadens bedeutend zunimmt [1, 48]. Durch eine begleitende Meniskus- oder Bandverletzung wird die Entstehung degenerativer Veränderungen zusätzlich gefördert [34]. Ursächlich hierfür ist nach heutiger Erkenntnis eine Verkleinerung der Last tragenden Fläche im Gelenk, die zu einer Überbeanspruchung des noch intakten Knorpels führt. Hierdurch wird sekundär die Synthese von entzündlichen Botenstoffen und katabolen Metalloproteinasen induziert und so die biochemische Knorpeldegeneration in Gang gesetzt.

\section{Resümee}

Knorpelschäden und Beeinträchtigungen von Gelenkbinnenstrukturen erhöhen das sekundäre Arthroserisiko. Möglicherweise haben primär umschriebene Knorpeldefekte einen deutlich höheren Anteil an der sekundären Arthroseentstehung als bisher angenommen.

\section{Therapieverfahren}

\section{Biologische Knorpelrekonstruktion}

Klinische und tierexperimentelle Studien haben gezeigt, dass eine frühzeitige biologische Rekonstruktion artikulärer Knorpelschäden einer rein konservativen Behandlung oder einer später durchgeführten biologischen Rekonstruktion in zweifacher Hinsicht überlegen ist:

- im Hinblick auf die eigentliche Defektausheilung und

- im Hinblick auf die Prävention von sekundär degenerativen Gelenkveränderungen $[22,43]$.

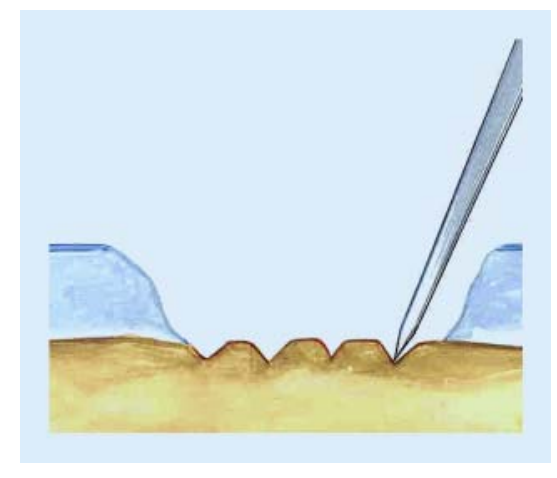

Abb. 1 ム Prinzip der Mikrofrakturierung 


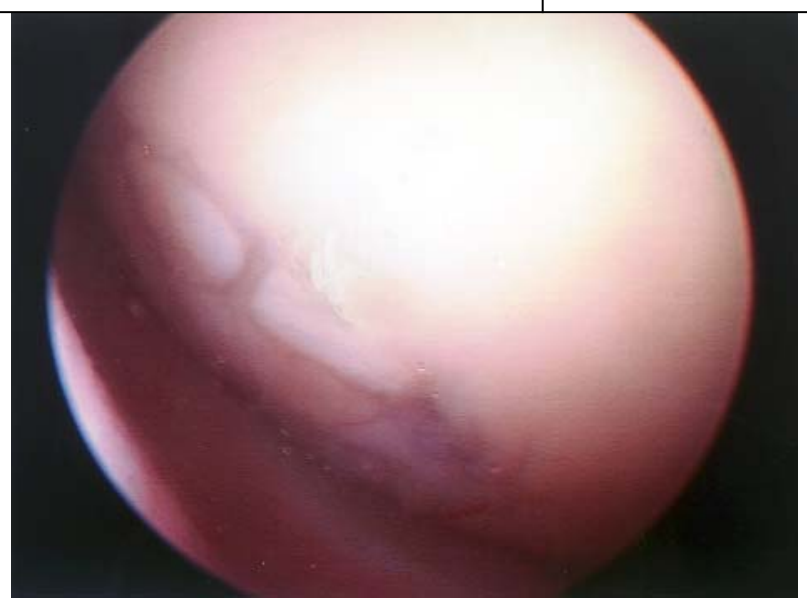

Abb. $2 \triangleleft$ Arthroskopischer Befund 1 Jahr nach autologer osteochondraler Transplantation

Die frühzeitige Defektsanierung, insbesondere von größeren, symptomatischen Knorpelschäden, scheint daher nicht nur aus therapeutischer, sondern auch aus sozioökonomischer Sicht sinnvoll [7]. In der Fachwelt hält jedoch die Diskussion darüber an, welche biologischen Rekonstruktionsverfahren bei klinisch relevanten Knorpelschäden vorzugsweise Anwendung finden sollten [45].

Aus einer plazebokontrollierten Studie mit prospektiv randomisiertem Design wurden für Débridement und Lavage des Kniegelenks enttäuschende klinische Resultate berichtet [36]. Beide Methoden können den zerstörten Gelenkknorpel nicht wieder aufbauen, weshalb sie als alleinige Techniken mit der Zielsetzung einer Rekonstruktion vollschichtiger Knorpelschäden auch nicht verwendet werden sollten.

Die kurz- bis mittelfristigen Resultate der reinen Transplantation von Periost oder Perichondrium sind überwiegend schlecht. Für beide Methoden sind hohe Revisions- und Komplikationsraten beschrieben [4, 10, 33]. Eine neuere tierexperimentellen Arbeit am Schaf konnte zeigen, dass die Verwendung von Periost zu einer erkennbaren Erhöhung der subchondralen Knochendichte führt [42]. Auch beim Menschen wurden nach Periost- oder Perichondriumtransplantationen Verknöcherungen des Regenerats beschrieben [10, 33].

Feingewebliche Untersuchungen von 22 Biopsien nach der Transplantation von Perichondrium beim Menschen ergaben, dass nur in 6 Fällen ein Gewebe entstand, in dem der Anteil von hyalinem Knorpel mehr als 50\% betrug [9]. Ähnlich unbefriedigende histologische Resultate wur- den nach Periosttransplantationen berichtet [4]. Das Risiko von Verknöcherungen und die insgesamt enttäuschenden histologischen Ergebnisse erklären die hohen Versagerquoten dieser Verfahren, weshalb sie heute nicht mehr empfohlen werden sollten $[7,20,33]$.

\section{Knochenmarkstimulation}

Nach sorgfältigem Débridement des geschädigten Knorpelgewebes wird bei knochenmarkstimulierenden Techniken, wie der Mikrofrakturierung, die subchondrale Knochenplatte mit kleinen Ahlen perforiert ( $\mathbf{A}$ Abb.1). Die iatrogene Eröffnung des Markraums führt im Defekt zur Bildung eines Zell-Blut-Koagels, dem so genannten "Super-Clot“, aus dem im weisatzgewebe entsteht. bewegen des Gelenks auf einer Motorschiene (CPM) soll diesen Prozess unterstützen [29]. Es gibt aber auch Arbeiten, die keine Verbesserung der klinischen Ergebnisse durch zusätzliche CPM-Behandlung nach Mikrofrakturierung gefunden haben [35].

Der Vorteil dieser Technik ist ihre einfache arthroskopische Durchführbarkeit. Jedoch erreicht das fibröse Narbengewebe, das nach einem solchen Verfahren im Normalfall entsteht, nicht die biomechanische Belastbarkeit des gesunden Gelenkknorpels. Außerdem sind im weiteren Verlauf auch hier Verknöcherungen beschrieben $[13,29]$.

In einer Studie aus den USA beschrieben $80 \%$ der Patienten ihre Beschwerden auch noch nach 7 Jahren als deutlich gebessert. Die Studienteilnehmer wurden teren Verlauf in der Regel ein fibröses Er-

Postoperativ kontinuierliches Durch- jedoch retrospektiv aus einem größeren Patientengut selektiert, und die durchschnittliche Defektgröße betrug lediglich $2,77 \mathrm{~cm}^{2}$ [47]. Die 10- bis 11-Jahres-Ergebnisse einer Studie nach Anbohrung bei geschlossenen Wachstumsfugen waren enttäuschend [10], wobei die Mikrofrakturierung der Anbohrung in den histologischen Ergebnissen möglicherweise überlegen sein könnte [32]. Unabhängig von der Defektgröße wiesen die Ergebnisse knochenmarkstimulierender Techniken auch eine gewisse Altersabhängigkeit auf. Bei Kindern und Jugendlichen waren sie meist gut [2], in der 4. Lebensdekade deutlich schlechter $[25,47]$.

Neuerdings hofft man, durch die zusätzliche Verwendung einer abdeckenden Matrix die Ergebnisse der Mikrofrakturierung zu verbessern. Eine tierexperimentelle Arbeit am Schaf konnte diesen Ansatz jedoch nicht bestätigen. 4 und 12 Monate postoperativ konnten zwischen den Ergebnissen der Mikrofrakturierung mit und ohne Matrixabdeckung keine signifikanten Unterschiede festgestellt werden. Die besten Resultate fanden sich in einer weiteren experimentellen Gruppe, in welcher das Biomaterial vor seiner Implantation zusätzlich mit autologen Chondrozyten besiedelt wurde [17].

Vermutlich ist bei Verwendung herkömmlicher Biomaterialien auch nicht zu erwarten, dass die bekannte Verknöcherungstendenz des Regenerats nach Mikrofrakturierung ausbleibt, da ein großer Anteil der stromalen Knochenmarkzellen [31] - ähnlich wie Periostzellen - eine osteogene Differenzierungsneigung hat.

In Zukunft lassen sich die klinischen und histologischen Ergebnisse der Mikrofrakturierung möglicherweise mit Hilfe intelligenter Biomaterialien (z. B. Matrizes plus integrierte Wachstums- und Differenzierungsfaktoren) verbessern, was Gegenstand künftiger Studien sein wird.

\section{Autologer osteochondraler Transfer}

Bei der im Bereich des Kniegelenks seit mehreren Jahren häufig angewandten Mosaikplastik bzw. OATS-Technik wird versucht, mit speziellen Instrumenten aus weniger belasteten Arealen der Femurkondylen Knorpel-Knochen-Zylinder $\mathrm{zu}$ entnehmen und press-fit in das vor- 
bereitete Empfängerlager im Defektbereich einzubringen. Die Ergebnisse dieser Methode (- Abb. 2) sind bei kleinen und mittleren Defekten auch nach längeren Verlaufszeiten überwiegend als gut zu bezeichnen [24]. Wegen der limitierten Spenderfläche und dem Risiko, dort eine Entnahmemorbidität zu induzieren, sowie der teilweise unbefriedigenden Oberflächenkongruenz ist die Anwendung der Mosaikplastik limitiert [30]. Die meisten Autoren empfehlen eine Beschränkung auf Defekte unter 3-4 $\mathrm{cm}^{2}$ Fläche [30, 24]. Zur Behandlung patellarer Knorpelschäden sollte die Mosaikplastik nach Meinung mancher Autoren grundsätzlich nicht mehr eingesetzt werden $[8,45]$.

Die Anwendung von allogenen Knorpel-Knochen-Transplantaten sollte wegen des Risikos einer Infektion oder Abstoßung auf Fälle beschränkt bleiben, bei denen autologe Verfahren nicht mehr möglich sind [21]. Wegen ihrer erheblichen Komorbidität gilt das Gleiche bezüglich der Indikation für den posterioren Femurkondylentransfer und die hieraus weiterentwickelte Methode der MegaOATS [14].

\section{Autologe Chondrozyten- transplantation (ACT)}

Sie wurde erstmals 1987 von einer schwedischen Arbeitsgruppe klinisch angewendet (s. Peterson et al. [37, 38, 39] und Brittberg et al. [12]). Patienteneigene Chondrozyten werden aus einer Knorpelbiopsie isoliert und in vitro vermehrt. Nach Anzucht einer ausreichenden Zellzahl werden sie unter einen wasserdicht aufgenähten Periostlappen injiziert [12]. Im weiteren Verlauf entsteht in den meisten Fällen ein Gewebe, das dem hyalinen Gelenkknorpel in seinen Eigenschaften sehr nahe kommt [11, 37, 41]. Auch nach langen Verlaufszeiten wurden von Peterson et al. $[38,39]$ überwiegend gute bis sehr gute klinische Ergebnisse berichtet, wobei es Hinweise auf eine Wechselbeziehung zwischen der Regeneration eines Knorpels mit hyalinen Eigenschaften $\mathrm{zu}$ guten klinischen Ergebnissen gibt [37]

\section{(• Abb. 3).}

Auch von anderen Arbeitsgruppen wurden inzwischen für den kurz- und mittelfristigen Verlauf überwiegend gute
Trauma Berufskrankh 2006 - 8 [Suppl 1]: S49-S54 DOI 10.1007/s10039-006-1115-1

(c) Springer Medizin Verlag 2006

\section{B. Schewe $\cdot$ K. Weise \\ Therapie von Knorpelschäden. Was ist gesichert?}

\section{Zusammenfassung}

Von allen großen Gelenken ist das Kniegelenk am häufigsten von einer Arthrose betroffen. Verschiedene Studien ergaben, dass Knorpeldefekte des Kniegelenks auch bei jüngeren Patienten nicht selten sind und dass mit zunehmender Defektgröße das sekundäre Arthroserisiko steigt. Um der Arthroseentstehung vorzubeugen, sollten klinisch symptomatische Knorpelschäden möglichst frühzeitig biologisch rekonstruiert und bestehende Achsfehlstellungen, Band- oder Meniskusverletzungen saniert werden. Erfolgen diese Rekonstruktionsmaßnahmen nicht, bleibt als alleinige Therapie bei einer dann fortgeschrit-

tenen Arthrose der endoprothetische Gelenkersatz. Für die biologische Rekonstruktion des Gelenkknorpels stehen verschiedene operative Verfahren zur Verfügung. Technik, Vor- und Nachteile sowie die mittel- bis langfristigen Ergebnisse der am häufigsten angewendeten Methoden werden in der vorliegenden Arbeit dargestellt, um deren Indikationsspektrum aufzuzeigen.

\section{Schlüsselwörter}

Gelenkknorpelschäden · Biologische Rekonstruktion - Indikationen - Operationstechnik . Endoprothetischer Gelenkersatz

\section{Treatment of cartilaginous damage. What is known for certain?}

\section{Abstract}

The knee is the large joint that is most frequently affected by osteoarthritis (OA). Several studies have shown that articular cartilage defects in the knee are far from rare, even in younger patients. There is also evidence that the risk of secondary osteoarthritis increases with extent of the primary defect. In order to avoid $O A$, treatment of symptomatic articular cartilage defects should be implemented as early as possible, to allow biological reconstruction and correction of any axis malalignment and injuries to ligaments or menisci. If this is not done OA will progress to the stage where the only possible treatment is insertion of an endoprosthesis. Various surgical techniques are available for biological reconstruction of the articular cartilage surface. This paper presents the techniques currently in use, their pros and cons and the medium- and long-term results of each to illustrate the indications appropriate to the different procedures.

\section{Keywords}

Articular cartilage defects - Biological reconstruction · Indications - Surgical techniques - Endoprosthetic joint replacement 


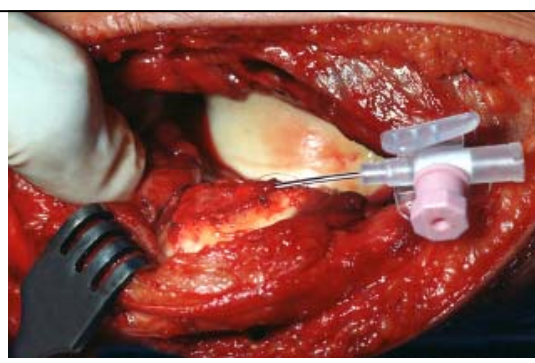

Abb. $3 \Delta$ „ "Klassische" ACT mit Periostlappendeckung an der Patella

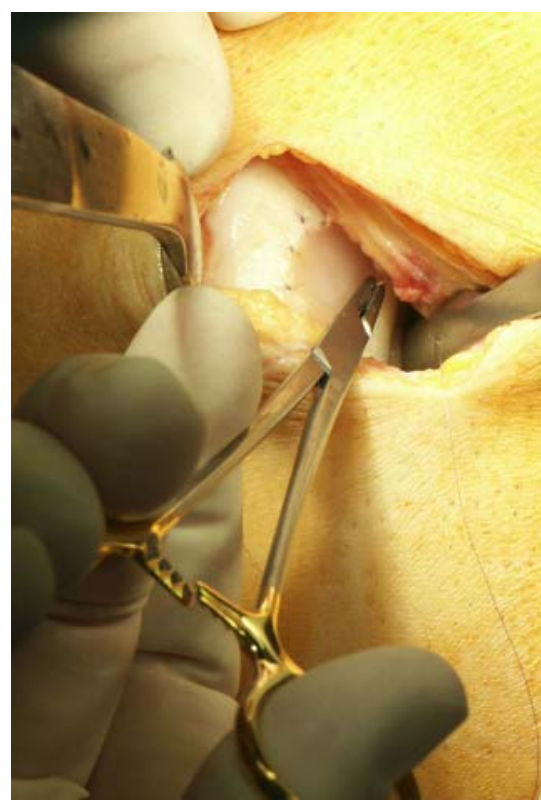

Abb. 4 \ Matrixgekoppelte ACT (NOVOCART ${ }^{\circ} 3 \mathrm{D}, \mathrm{B} \mid$ Braun Aesculap, Tuttlingen)

Ergebnisse beschrieben [20, 26, 45]. Mit der ACT können selbst großflächige Defekte erfolgreich behandelt werden [37, 39].

\section{Vergleichende Studien}

Vergleichende MR-Untersuchungen mit 112 Patienten (86-mal Mikrofrakturierung und 35-mal ACT) konnten für die ACT im Vergleich zur Mikrofrakturierung zu allen Zeitpunkten eine bessere Defektauffüllung zeigen, wobei in der Gruppe der Mikrofrakturierung oft Verknöcherungen im Defekt und ein progredienter Regeneratverlust auftraten [13]. Ähnliche Ergebnisse wurden aus einer anderen klinischen Studie berichtet, in der eine matrixgekoppelte Form der ACT mit der Mikrofrakturierung verglichen wurde [6].

In einer multizentrischen Studie aus Norwegen mit 8o Patienten wurden nach 2 Jahren Verlaufszeit nur geringe Unter-

\section{Aktuelle Themen}

schiede zwischen den klinischen Ergebnissen der Mikrofrakturierung und der ACT gefunden. Ein Zusammenhang zwischen den histologischen und klinischen Befunden konnte nicht festgestellt werden. Tendenziell ergaben sich für die ACT bessere histologische Ergebnisse, wobei allerdings die Patientenzahl für eine statistische Signifikanz zu klein war [32]. Des Weiteren muss aufgrund der bisherigen Erkenntnisse zur Transplantatreifung nach ACT angenommen werden, dass die Biopsieentnahme im postoperativen Verlauf zu früh stattfand, da das entstehende Gewebe einem längeren Umbauvorgang (Remodeling) unterliegt [41]. Eine ähnlich zeitabhängige Verbesserung der Ergebnisse wurde auch von anderen Autoren nach ACT beschrieben [26].

In der bereits erwähnten Studie aus Norwegen zeigten sich klinisch in der Gruppe der Patienten mit Mikrofrakturierung für Defekte unter $4 \mathrm{~cm}^{2}$ signifikant bessere Ergebnisse als bei größeren Defekten. Bei den ACT-Patienten wurde jedoch keine derartige Korrelation zwischen der Defektgröße und dem klinischen Ergebnis beschrieben [32].

Eine prospektiv kontrollierte Multicenterstudie mit Defekten $>2 \mathrm{~cm}^{2}$ und Beobachtungszeiten von über 6 Jahren ergab für die ACT im Vergleich zur Mikrofrakturierung $\mathrm{z}$. T. signifikant bessere klinische Ergebnisse [3].

In einer prospektiv randomisierten Studie zur ACT vs. Mosaikplastik [28] mit jeweils 2o Patienten pro Gruppe und einer durchschnittlichen Defektgröße von $3,86 \mathrm{~cm}^{2}$ wurden nach 2 Jahren keine wesentlichen Unterschiede festgestellt.

Eine weitere prospektiv randomisierte Studie zur selben Fragestellung, jedoch mit 100 Patienten und einer durchschnittlichen Defektgröße von $4,66 \mathrm{~cm}^{2}$, ergab für die ACT signifikant bessere Resultate als für die Mosaikplastik. Bei den Patienten mit Letzterer wurden nach durchschnittlich 19 Monaten bereits häufig die ersten Zeichen degenerativer Veränderungen beobachtet, bei den ACT-Patienten jedoch nicht [8].

\section{Resümee}

Aufgrund der vorliegenden Studienergebnisse muss man derzeit davon ausgehen, dass die ACT nach dem Schluss der
Wachstumsfugen v. a. bei größeren Knorpelschäden und nach längeren Verlaufszeiten den anderen hier beschriebenen Methoden zur biologischen Knorpelrekonstruktion überlegen ist. Daher sollte bei traumatischen Knorpelschäden oder Defekten durch Osteochondrosis dissecans mit bestehender klinischer Symptomatik und einer Flächenausdehnung $>3-4 \mathrm{~cm}^{2}$ beim Erwachsenen die ACT als primäres Behandlungsverfahren indiziert werden. Bei kleineren Knorpelschäden ist zunächst die Mikrofrakturierung und bei mittleren Defektgrößen die Mosaikplastik zu empfehlen. Bei Kindern und Jugendlichen sollten aufgrund der intrinsischen Regenerationsfähigkeit - unabhängig von der Defektgröße - primär knochenmarkstimulierende Techniken zumindest versucht werden $[7,20]$, bevor zu invasiveren Maßnahmen gegriffen wird.

\section{Trägergekoppelte ACT}

Trotz ihrer überwiegend guten klinischen Ergebnisse weist die konventionelle Form der ACT auch Schwächen auf. Ihre häufigste Komplikation ist die Transplantathypertrophie [37], die nach derzeitigem Kenntnisstand ihre Ursache im verwendeten Periostlappen hat [23].

Bei frühzeitiger Delamination des Knochenhautlappens oder unzureichender Zellqualität kann es zu einer Defektauffüllung mit qualitativ minderwertigen Ersatzgewebe oder sogar zum vollständigen Transplantatversagen kommen. Für die Periostentnahme und die wasserdichte Aufnaht des Knochenhautlappens ist in der Regel eine größere Arthrotomie erforderlich, die in manchen Fällen mit entsprechenden postoperativen Beschwerden verbunden sein kann. Bei unvollständiger oder fehlender Knorpelschulter lässt sich der Knochen-HautLappen oft nur schwer, manchmal auch gar nicht über den Defekt nähen [7].

Aufgrund der genannten Probleme finden in letzter Zeit vermehrt Biomaterialien als Träger für die Chondrozyten Verwendung. Im Wesentlichen werden Trägermaterialien aus unterschiedlichen Kollagenen, synthetischen Polymeren oder aus veresterter Hyaluronsäure verwendet. Entscheidend ist, dass das Biomaterial die physiologische Umgebung der Chondro- 
zyten möglichst genau imitiert und diese biochemisch nicht irritiert. Das Trägermaterial sollte die Knorpelzellen also nicht wesentlich stören, denn die Gewebereparatur geht auch bei der trägergekoppelten Form der ACT von den transplantierten Chondrozyten aus. Das Trägermaterial wird im Therapieverlauf wieder resorbiert. Auch unter Verwendung eines Biomaterials sind an die zu transplantierenden Chondrozyten dieselben Qualitätsanforderungen zu stellen, wie sie die Fachgesellschaften für die klassische ACT empfohlen haben [7].

Bei einer trägergekoppelten Chondrozytentransplantation entfallen die Entnahme und Aufnaht eines Periostlappens. Das Risiko der Transplantathypertrophie sinkt, die Operationszeit verkürzt sich wesentlich, und der operative Zugang kann kleiner gehalten werden. Da auch eine wasserdichte Naht des Periostlappens entfällt, können unter Verwendung eines Trägermaterials mühelos Defekte ohne vollständig erhaltene Knorpelschulter versorgt werden.

In unserem Haus wird seit Ende 2003 die matrixgekoppelte Chondrozytentransplantation (NOVOCART 3 D, B $\mid$ Braun Aesculap, Tuttlingen) durchgeführt (- Abb. 4). Die ersten klinischen Ergebnisse sind viel versprechend. Die Frage, ob diese Form der ACT der „klassischen“ Methode mit Verwendung des Periostlappens letztlich auch nach längeren Verlaufszeiten im klinischen Ergebnis überlegen ist, wird derzeit im Rahmen einer Nachuntersuchung geklärt.

\section{Zukunftsperspektiven}

Das wissenschaftliche Verständnis der Ätiologie von Knorpelschäden und der Biologie des hyalinen Knorpels hat in den letzten Jahren deutlich zugenommen. So ist mittlerweile bekannt, dass eine ganze Reihe verschiedener Aspekte das Regenerationsvermögen des Gelenkknorpels beeinflusst. Hierbei kommt insbesondere auch bestimmten Wachstums- und Differenzierungsfaktoren eine zentrale Bedeutung zu.

Unsere Arbeitsgruppe ist an der Entwicklung der nächsten Generation von Biomaterialien beteiligt. Diese auch als intelligente Biomaterialien („smart bio- materials") bezeichneten Matrizes können gezielt Milieuveränderungen in vivo und entwicklungsabhängige Bedürfnisse transplantierter Zellen während der Regenerationsphase berücksichtigen. Auf dieser Basis ist es denkbar, in Zukunft möglicherweise auch fortgeschrittene Knorpelschäden beim älteren Patienten biologisch $\mathrm{zu}$ rekonstruieren. Ferner erlauben diese neuartigen Matrizes nach unserer bisherigen Erkenntnis eine gezielte und stabile chondrogene Differenzierung von Zellen im Rahmen einer Mikrofrakturierung.

Die Verwendung intelligenter Biomaterialien würde somit zu einer erheblichen Vereinfachung und Kostenreduktion in der Versorgung lokal begrenzter Knorpelschäden beitragen.

\section{Korrespondierender Autor Dr. B. Schewe \\ BG-Unfallklinik \\ Schnarrenbergstraße 95, 72076 Tübingen \\ bschewe@bgu-tuebingen.de}

Interessenkonflikt. Es besteht kein Interessenkonflikt. Der korrespondierende Autor versichert, dass keine Verbindungen mit einer Firma, deren Produkt in dem Artikel genannt ist, oder einer Firma, die ein Konkurrenzprodukt vertreibt, bestehen. Die Präsentation des Themas ist unabhängig und die Darstellung der Inhalte produktneutral.

\section{Literatur}

1. Aglietti P, Ciardullo A, Giron F et al. (2001) Results of arthroscopic excision of the fragment in the treatment of osteochondritis dissecans of the knee. Arthroscopy 17: 741-746

2. Anderson AF, Richards DB, Pagnani MJ et al. (1997) Antegrade drilling for osteochondritis dissecans of the knee. Arthroscopy 13: 319-324

3. Anderson AF, Fu FH, Mandelbaum B et al. (2003) A controlled study of autologous chondrocyte implantation versus microfracture for articular cartilage lesions of the femur. Transactions of the 70th Annual Meeting of the American Academy of Orthopaedic Surgeons 2003, New Orleans, USA; February 5-9, 2003

4. Angermann P, Riegels-Nielsen P, Pedersen H (1998) Osteochondritis dissecans of the femoral condyle treated with periosteal transplantation. Poor outcome in 14 patients followed for 6-9 years. Acta Orthop Scand 69: 595-597

5. Aroen A, Loken S, Heir S et al. (2004) Articular cartilage lesions in 993 consecutive knee arthroscopies. Am J Sports Med 32: 211-215

6. Bachmann G, Basad E, Lommel D et al. (2004) Die MRT in der Verlaufskontrolle nach matrixgestützer autologer Chondrozytenimplantation ( $\mathrm{MACl}^{\circ}$ ) und Mikrofrakturierung. Radiologe 44: 773-782
7. Behrens P, Bosch U, Bruns J et al. (2004) Indikations- und Durchführungsempfehlungen der Arbeitsgemeinschaft "Geweberegeneration und Gewebeersatz" zur Autologen Chondrozyten-Transplantation (ACT). Z Orthop Ihre Grenzgeb 142: 529-539

8. Bentley G, Biant LC, Carrington RW et al. (2003) A prospective randomised comparison of autologous chondrocyte implantation versus mosaicplasty for osteochondral defects in the knee. J Bone Joint Surg Br 85: 223-230

9. Bouwmeester $P$, Kuijer R, Terwindt-Rouwenhorst $E$ et al. (1999) Histological and biochemical evaluation of perichondrial transplants in human articular cartilage defects. J Orthop Res 17: 843-849

10. Bouwmeester PS, Kuijer R, Homminga GN et al. (2002) A retrospective analysis of two independent prospective cartilage repair studies: autogenous perichondrial grafting versus subchondral drilling. 10 years follow up. J Orthop Res 20: 267273

11. Briggs TW, Mahroof S, David LA et al. (2003) Histological evaluation of chondral defects after autologous chondrocyte implantation of the knee. J Bone Joint Surg Br 85: 1077-1083

12. Brittberg M, Lindahl A, Nilsson A et al. (1994) Treatment of deep cartilage defects in the knee with autologous chondrocyte transplantation. N Engl J Med 331: 889-895

13. Brown WE, Potter HG, Marx RG et al. (2004) Magnetic resonance imaging appearance of cartilage repair in the knee. Clin Orthop 422: 214-223

14. Brucker P, Agneskircher JD, Burkart A et al. (2002) Mega-OATS: Technik und Ergebnisse. Unfallchirurg 105: 443-449

15. Buckwalter JA (2002) Articular cartilage injuries. Clin Orthop 402: 21-37

16. CurlWW, Krome J, Gordon ES et al. (1997) Cartilage injuries: a review of 31,516 knee arthroscopies. Arthroscopy 13: 456-460

17. Dorotka R, Bindreiter U, Macfelda K et al. (2005) Marrow stimulation and chondrocyte transplantation using a collagen matrix for cartilage repair. Epub ahead of print. Osteoarthritis Cartilage 13: 655-664

18. Engelhardt M (2003) Epidemiologie der Arthrose in Westeuropa. Dtsch Z Sportmed 54: 171-175

19. Felson DT (2004) Risk factors for osteoarthritis: understanding joint vulnerability. Clin Orthop Relat Res [Suppl] 427: 16-21

20. Gaissmaier C, Fritz J, Mollenhauer J et al. (2003) Verlauf klinisch symptomatischer Knorpelschäden des Kniegelenks: Ergebnisse ohne und mit biologischer Rekonstruktion. Dtsch Arztebl 100: A24482453

21. Gross AE (2002) Repair of cartilage defects in the knee. J Knee Surg 15: 167-169

22. Gudas R, Kunigiskis K, Kalensinskas RJ (2002) Long-term follow-up of osteochondritis dissescans. Medicina 38: 284-288

23. Haddo O, Mahroof S, Higgs D et al. (2004) The use of chondrogide membrane in autologous chondrocyte implantation. Knee 11: 51-55

24. Hangody L, Fules P (2003) Autologous osteochondral mosaicplasty for the treatment of fullthickness defects of weight-bearing joints: ten years of experimental and clinical experience. J Bone Joint Surg Am [Suppl 2] 85: 25-32

25. Hankemeier S, Müller EJ, Kaminski A et al. (2003) 10-Jahres-Ergebnisse knochenmarkstimulierender Therapie der Osteochondrosis dissecans tali. Unfallchirurg 106: 461-466 
26. Henderson I, Francisco R, Oakes B et al. (2005) Autologous chondrocyte implantation for treatment of focal chondral defects of the knee - a clinical, arthroscopic, MRI and histologic evaluation at 2 years. Knee 12: 209-216

27. Hjelle K, Solheim E, Strand T et al. (2002) Articular cartilage defects in 1.000 knee arthroscopies. Arthroscopy 18: 730-734

28. Horas U, Pelinkovic D, Herr G et al. (2003) Autologous chondrocyte implantation and osteochondral cylinder transplantation in cartilage repair of the knee joint: a prospective, comparative trial. J Bone Joint Surg Am 85: 185-192

29. Hunziker EB (2002) Articular cartilage repair: basic science and clinical progress. A review of the current status and prospects. Osteoarthritis Cartilage 10: 432-463

30. Jakob RP, Franz T, Gautier E et al. (2002) Autologous osteochondral grafting in the knee: indication, results, and reflections. Clin Orthop 401: 170 184

31. Kassem M, Risteli L, Mosekilde L et al. (1991) Formation of osteoblast-like cells from human mononuclear bone marrow cultures. APMIS 99: 269-274

32. Knutsen G, Engebretsen L, Ludvigsen TC et al. (2004) Autologous chondrocyte implantation compared with microfracture in the knee - a randomized trial. J Bone Joint Surg Am 86: 455-464

33. Madsen BL, Noer HH, Carstensen JP (2000) Longterm results of periosteal transplantation in osteochondritis dissecans of the knee. Orthopedics 23: 223-226

34. Maletius W, Messner K (1996) Chondral damage and age depress the long-term prognosis after partial meniscectomy. A 12- to 15-year followup study. Knee Surg Sports Traumatol Arthrosc 3: 211-214

35. Marder RA, Hopkins G Jr, Timmerman LA (2005) Arthroscopic microfracture of chondral defects of the knee: a comparison of two postoperative treatments. Arthroscopy 21: 152-158

36. Moseley JB, O'Malley K, Petersen NJ (2002) A controlled trial of arthroscopic surgery for a osteoarthritis of the knee. N Engl J Med 347: 81-88

37. Peterson L, Minas T, Brittberg M et al. (2000) Twoto 9-year outcome after autologous chondrocyte transplantation of the knee. Clin Orthop 374: 212234

38. Peterson L, Brittberg M, Kiviranta I et al. (2002) Autologous chondrocyte transplantation. Biomechanics and long-term durability. Am J Sports Med 30: 2-12

39. Peterson L, Minas T, Brittberg M et al. (2003) Treatment of osteochondritis dissecans of the knee with autologous chondrocyte transplantation: results at two to ten years. J Bone Joint Surg Am 85 [Suppl 2]: 17-24

40. Pientka L (2000) Arthrose als Volkskrankheit. J Clin Res 6 [Suppl 1]: 11-13

41. Roberts S, Hollander AP, Caterson B et al. (2001) Matrix turnover in human cartilage repair tissue in autologous chondrocyte implantation. Arthritis Rheum 44: 2586-2598

42. Russlies M, Behrens P, Ehlers EM et al. (2005) Periosteum stimulates subchondral bone densification in autologous chondrocyte transplantation in a sheep model. Cell Tissue Res 319: 133-142

43. Saris DB, Dhert WJ, Verbout AJ (2003) Joint homeostasis. The discrepancy between old and fresh defects in cartilage repair. J Bone Joint Surg Br 85: 1067-1076
44. Shelbourne KD, Jari S, Gray T (2003) Outcome of untreated traumatic articular cartilage defects of the knee: a natural history study. J Bone Joint Surg Am 85 [Suppl 2]: 8-16

45. Smith GD, Knutsen G, Richardson JB (2005) A clinical review of cartilage repair techniques. J Bone Joint Surg Br 87: 445-449

46. Spector TD, Hart DJ, Leedham-Green M (1991) The prevalence of knee and hand osteoarthritis $(O A)$ in the general population using different clinical criteria: the Chingford study. Arthritis Rheum 34: 171

47. Steadman JR, Briggs KK, Rodrigo JJ et al. (2003) Outcomes of microfracture for traumatic chondral defects of knee: average 11-year follow-up. Arthroscopy 19: 477-484

48. Twyman RS, Desai K, Aichroth PM (1991) Osteochondritis dissecans of the knee. A long term study. J Bone Joint Surg Br 73: 461-464 